\title{
@creative
}

ISSN 1855-3966 (printed edn.), ISSN 1855-3974 (electronic edn.)

\author{
ARS MATHEMATICA CONTEMPORANEA 15 (2018) 487-497 \\ https://doi.org/10.26493/1855-3974.1359.b33 \\ (Also available at http://amc-journal.eu)
}

\section{The isolated-pentagon rule and nice substructures in fullerenes*}

\author{
Hao Li \\ Laboratoire de Recherche en Informatique, UMR 8623, C.N.R.S.-Université Paris-sud, \\ F-91405, Orsay, France \\ Heping Zhang ${ }^{\dagger}$ \\ School of Mathematics and Statistics, Lanzhou University, \\ Lanzhou, Gansu 730000, P.R. China
}

Received 22 March 2017, accepted 6 September 2017, published online 5 September 2018

\begin{abstract}
After fullerenes were discovered, Kroto in 1987 proposed first the isolated-pentagon rule (IPR): the most stable fullerenes are those in which no two pentagons share an edge, that is, each pentagon is completely surrounded by hexagons. To now the structures of the synthesized and isolated (neutral) fullerenes meet this rule. The IPR can be justified from local strain in geometry and $\pi$-electronic resonance energy of fullerenes. If two pentagons abut in a fullerene, a 8-circuit along the perimeter of the pentalene (a pair of abutting pentagons) occurs. This paper confirms that such a 8-circuit is always a conjugated cycle of the fullerene in a graph-theoretical approach. Since conjugated circuits of length 8 destabilize the molecule in conjugated circuit theory, this result gives a basis for the IPR in $\pi$-electronic resonance. We also prove that each 6-circuit (hexagon) and each 10-circuit along the perimeter of a pair of abutting hexagons are conjugated. Two such types of conjugated circuit satisfy the $(4 n+2)$-rule, and thus stabilise the molecule.
\end{abstract}

Keywords: Fullerene, patch, stability, isolated pentagon rule, Kekulé structure, conjugated cycle, cyclic edge-cut.

Math. Subj. Class.: 05C70, 05C10, 92E10

* This work was supported by NSFC (Grant Nos. 11371180, 11871256).

${ }^{\dagger}$ Corresponding author.

E-mail addresses: Hao.Li@1ri.fr (Hao Li), zhanghp@1zu.edu.cn (Heping Zhang)

()( T) This work is licensed under https://creativecommons.org/licenses/by/3.0/ 


\section{Introduction}

The fullerenes are closed carbon-cage molecules such that every carbon atom has bonds to three other atoms, and the length of each carbon ring is either 5 or 6 . Ever since the first fullerene, Buckministerfullerene $\mathrm{C}_{60}$, was discovered by Kroto et al. in 1985 [15], the stabilities of fullerenes have attracted many theorist's attentions. The simple Hückel molecular orbital model that predicts reliably the relative stabilities of planar aromatic hydrocarbons is not generally found to work so well for fullerenes. Kroto [14] in 1987 proposed first the isolated-pentagon rule (IPR): the most stable fullerenes are those in which no two pentagons share an edge, that is, each pentagon is completely surrounded by hexagons. Schmalz et al. [23] gave a more theoretical discussion of the rule in support of the fullerene hypothesis. Indeed the structures of the synthesized and isolated fullerenes meet this rule. The IPR can be justified from local strain and $\pi$-electronic resonance of fullerenes; for details, also see a book due to Fowler and Manolopoulos [7]. Pentagon adjacency leads to higher local curvature of the molecule surface and increases the strain energy. On the other hand, according to Hückel $(4 n+2)$-rule, conjugated circuits of length $6,10,14, \ldots$ stabilize the molecule, whereas conjugated circuits of length $4,8,12, \ldots$ destabilize the molecule. Here a conjugated circuit is a cycle of alternating single and double bonds within a Kekulé structure. If two pentagons abut in a fullerene, the conjugated or resonant 8-circuit along the perimeter of the pentalene may occur, and this leads to resonance destabilization [22]. This is an interpretation of IPR in $\pi$-electronic resonation stabilization. However, a problem occurs: In a fullerene, is every 8-length circuit conjugated? To now we have not seen any definite answer to this problem in mathematics. In this article we investigate nice patches of a fullerene by applying some small cyclic edge-cuts of graphs and present a positive answer to the above problem (a patch of a fullerene is nice if its Kekule structure can be extended to a Kekulé structure of the entire fullerene). As immediate consequences of our main theorems, we have that every 8-length circuit of a fullerene surrounds a pentalene (a pair of abutting pentagons) and is conjugated or alternating with respect to a Kekulé structure (see Corollary 3.4). This confirms the destabilization of any pentalene as a nice substructure to the entire fullerene and thus gives a mathematical support for the IPR of fullerenes. Furthermore we also show that in a fullerene every hexagon is a conjugated 6-circuit (see Corollary 3.3) and the boundary along a naphthalene (i.e. a pair of abutting hexagons) is a conjugated 10-circuit (see Corollary 4.2). The former has already been proved (see [26]). In conjugated circuit theory [10, 19, 20], conjugated 6-circuits and 10circuits contribute stabilizations of fullerenes and the small conjugated circuits have the greatest effects (positive and negative) on stability. For recent discussions on the IPR of fullerenes about steric strain factor and $\pi$-electronic resonance factor, see $[1,2,8,13,21]$. For mathematical aspects of fullerenes, see a recent survey [3].

\section{Preliminary}

To obtain the above end we now start our arguments in a graph-theoretical approach. As a molecular graph of a fullerene, a fullerene graph is a 3-connected planar cubic graph with only pentagonal and hexagonal faces. It is well known that a fullerene graph on $n$ vertices exists for every even $n \geq 20$ except $n=22$ [9]. By Euler's polyhedron formula, every fullerene graph with $n$ vertices has exactly 12 pentagonal faces and $(n / 2-10)$ hexagonal faces.

Let $G$ be a graph with vertex-set $V(G)$ and edge-set $E(G)$. An edge set $M$ of a graph 
$G$ is called a matching if no two edges in $M$ have a common endvertex. A matching $M$ of $G$ is perfect if every vertex of $G$ is incident with one edge in $M$. In organic molecular graphs, perfect matchings correspond to Kekulé structures, playing an important role in analysis of the resonance energy and stability of polycyclic aromatic hydrocarbons.

The following classical theorem is Tutte's 1-factor theorem on the existence of perfect matching of a graph [24]. For detailed monograph on matching theory, see Lovász and Plummer [17].

Theorem 2.1. A graph $G$ has a perfect matching if and only if odd $(G-S) \leq|S|$ for each $S \subseteq V(G)$, where odd $(G-S)$ denotes the number of odd components in subgraph $G-S$.

Subgraph $G^{\prime}$ of a graph $G$ is called nice if $G-V\left(G^{\prime}\right)$ has a perfect matching. In particular, an even cycle $C$ of a graph $G$ is nice if $G$ has a perfect matching $M$ such that $C$ is an $M$-alternating cycle, i.e. the edges of $C$ alternate in $M$ and $E(G) \backslash M$. A nice even cycle is also called resonant or conjugated cycle (or circuit) in chemical literature. For convenience, a cycle of length $k$ is said to be a $k$-cycle or $k$-circuit.

For nonempty subsets $X, Y$ of $V(G)$, let $[X, Y]$ denote the set of edges of $G$ that each has one end-vertex in $X$ and the other in $Y$. If $\bar{X}=V(G) \backslash X \neq \emptyset$, then $\nabla(X):=[X, \bar{X}]$ is called an edge-cut of $G$, and $k$-edge-cut whenever $|[X, \bar{X}]|=k$. The edges incident with a single vertex form a trivial edge-cut. For a subgraph $H$ of $G$, let $\bar{H}:=G-V(H)$. We simply write $\nabla(H)$ for $\nabla(V(H))$.

Lemma 2.2 ([25]). Every 3-edge-cut of a fullerene graph is trivial.

Lemma 2.3 ([25]). Every 4-edge-cut of a fullerene graph isolates an edge.

An edge-cut $S=\nabla(X)$ of $G$ is cyclic if at least two components of $G-S$ each contains a cycle. The minimum size of cyclic edge-cuts of $G$ is called cyclic edge-connectivity of $G$, denoted by $c \lambda(G)$.

Theorem 2.4 ([6, 12, 18]). Let $F$ be any fullerene graph. Then $c \lambda(F)=5$.

From the definition with the above properties we know that each fullerene graph has the girth 5 (the minimum length of cycles), and each of its 5 -cycles and 6 -cycles bounds a face. A cyclic $k$-edge-cut of a graph isolating just a $k$-cycle will be called trivial.

Theorem 2.5 ([12, 16]). A fullerene graph with a non-trivial cyclic 5-edge-cut is a nanotube with two disjoint pentacaps (see Figure 1), and each non-trivial cyclic 5-edge-cut must be an edge set between two consecutive concentric cycles of length 10.

A fullerene patch is a 2-connected plane graph with all faces pentagonal or hexagonal except one external face, all internal vertices (not incident with the external face) of degree 3 and those incident with the external face having degree 2 or 3 . The cycle bounding the external face is the boundary of the patch. We can count the pentagons of a fullerene patch as internal faces as follows.

Lemma 2.6 ([4]). For fullerene patch $G$, let $p_{5}$ denote the number of pentagonal faces other than the external face. Then

$$
p_{5}=6+k_{3}-k_{2}=6+2 k_{3}-l,
$$

where $k_{2}$ and $k_{3}$ denote the number of vertices of degree 2 and 3 on the boundary of $G$, respectively, and $l$ is the boundary length. 


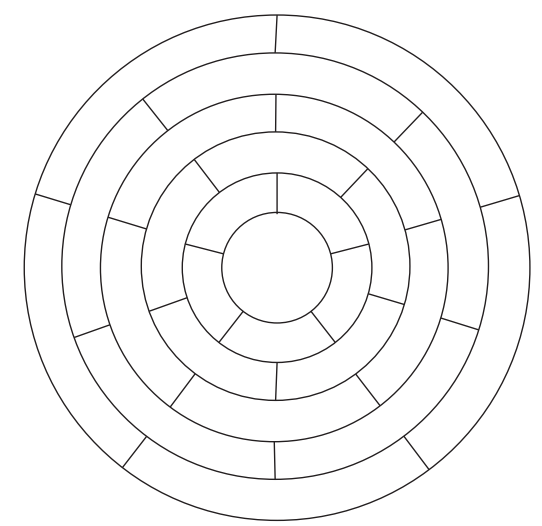

Figure 1: A fullerene with a non-trivial cyclic 5-edge-cut.

For $T \subseteq V(G)$, the induced subgraph of $G$ by $T$ consists of $T$ and all edges whose endvertices are contained in $T$, denoted by $G[T]$.

In the next two sections we will investigate nice patches of fullerene graphs in cyclic 6 -edge-cut and 8-edge-cut cases, respectively.

\section{Cyclic 6-edge-cut}

We first consider a more general case than fullerene patches.

Theorem 3.1. Let $F_{0}$ be a connected induced subgraph of a fullerene graph $F$ such that interior faces of $F_{0}$ exist and each one is a pentagon or hexagon. If $F$ has exactly six edges from $F_{0}$ to the outside $\bar{F}_{0}=F-V\left(F_{0}\right)$, then $F_{0}$ has a perfect matching.

Proof. Let $n_{0}$ and $\epsilon_{0}$ denote the numbers of vertices and edges of $F_{0}$ respectively. Then $3 n_{0}=2 \epsilon_{0}+6$, which implies that $n_{0}$ is even, i.e. $F_{0}$ has an even number of vertices.

We will prove that $F_{0}$ has a perfect matching by Tutte's theorem. To the contrary suppose that $F_{0}$ has no perfect matchings. By Theorem 2.1, there exists a subset $X_{0} \subset$ $V\left(F_{0}\right)$ such that

$$
\operatorname{odd}\left(F_{0}-X_{0}\right)>\left|X_{0}\right|
$$

For the sake of convenience, let $\alpha:=\operatorname{odd}\left(F_{0}-X_{0}\right)$. Since $\alpha$ and $\left|X_{0}\right|$ have the same parity, we have

$$
\alpha \geq\left|X_{0}\right|+2 \text {. }
$$

Let $G_{1}, \ldots, G_{\alpha}$ and $G_{\alpha+1}, \ldots, G_{\alpha+\beta}$ denote respectively the odd components and the even components of $F_{0}-X_{0}$, where $\beta$ denotes the number of even components of $F_{0}-X_{0}$. For $i=1,2, \ldots, \alpha+\beta$, let $m_{i}$ denote the number of edges of $F_{0}$ which are sent to $X_{0}$ from $G_{i}$, and $\gamma_{i}$ (resp. $\gamma_{0}$ ) the number of edges of $F$ from $G_{i}$ (resp. $\left.X_{0}\right)$ to $\bar{F}_{0}$. Since $\nabla\left(F_{0}\right)$ is a 6 -edge-cut of $F$, we have

$$
\left|\nabla\left(F_{0}\right)\right|=\sum_{i=0}^{\alpha+\beta} \gamma_{i}=6 .
$$

Since $F$ is 3 -connected, for $i=1, \ldots, \alpha, \ldots, \alpha+\beta$ we have

$$
\left|\nabla\left(G_{i}\right)\right|=m_{i}+\gamma_{i} \geq 3 \text {. }
$$




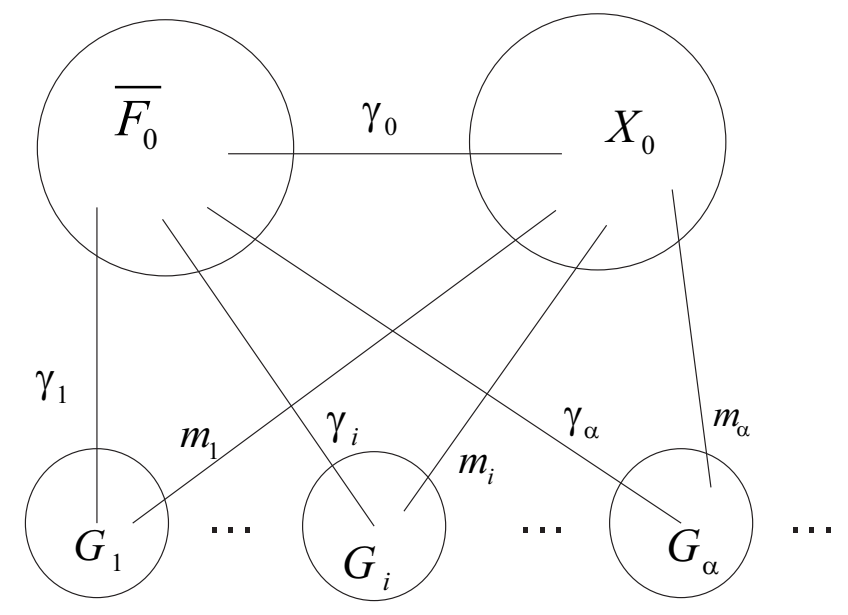

Figure 2: Illustration for the proof of Theorem 3.1.

By taking the number of edges of $F$ from the components $G_{i}$ to $\bar{F}_{0}$ and $X_{0}$ into account and by using Equation (3.3) and Inequalities (3.2) and (3.4) we have

$$
\begin{aligned}
3(\alpha+\beta) & \leq \sum_{i=1}^{\alpha+\beta}\left(m_{i}+\gamma_{i}\right) \\
& \leq 3\left|X_{0}\right|-\gamma_{0}+\sum_{i=1}^{\alpha+\beta} \gamma_{i} \\
& =3\left|X_{0}\right|+6-2 \gamma_{0} \\
& \leq 3 \alpha-2 \gamma_{0}
\end{aligned}
$$

which implies that $\beta=0, \gamma_{0}=0$ and equalities always hold. Hence $\sum_{i=1}^{\alpha} \gamma_{i}=6$, and $\alpha=\left|X_{0}\right|+2$. Further, the second equality in (3.5) implies that $X_{0}$ is an independent set of $F_{0}$. The first equality in (3.5) implies that $m_{i}+\gamma_{i}=3$ for each $1 \leq i \leq \alpha$, that is, $\nabla\left(G_{i}\right)$ is a 3-edge-cut of $F$. So by Lemma 2.2 it is a trivial edge-cut and each $G_{i}$ is a singleton. Let $Y_{0}$ denote the set of all singletons $G_{i}$. Then $F_{0}$ is a bipartite graph with partite sets $X_{0}$ and $Y_{0}$.

If $F_{0}$ has no vertices of degree one, then $F_{0}$ is 2-connected. Otherwise, $F_{0}$ has a bridge, the deletion of which results in two components each containing a cycle. So the bridge together with at most three edges in $\nabla\left(F_{0}\right)$ form a cyclic edge-cut, contradicting that $c \lambda(F)=5$ (Theorem 2.4). Hence $F_{0}$ is a fullerene patch. Since $k_{2}=\left|\nabla\left(F_{0}\right)\right|=6$, by Lemma 2.6 we have that the number $p_{5}$ of pentagons contained in $F_{0}$ is equal to the number $k_{3}$ of vertices of degree three lying on the boundary of $F_{0}$. Since $F_{0}$ is bipartite, $k_{3}=p_{5}=$ 0 , which implies that $F_{0}$ is just a hexagon, contradicting that $\alpha=\left|Y_{0}\right|=\left|X_{0}\right|+2$.

If $F_{0}$ has a vertex $x$ of degree one, let $x y$ be the edge of $F_{0}$, and $x y_{1}$ and $x y_{2}$ be the other two edges in $F$ incident with $x$. Then $\nabla\left(F_{0}-x\right)=\left(\nabla\left(F_{0}\right) \backslash\left\{x y_{1}, x y_{2}\right\}\right) \cup\{x y\}$ forms a cyclic 5 -edge-cut of $F$ since $F_{0}-x$ contains all cycles of $F_{0}$ and $\overline{F_{0}-x}$ can be obtained from $F-F_{0}$ by adding a 2-length path $y_{1} x y_{2}$ and contains at least seven pentagons. Since $F_{0}-x$ is bipartite, cyclic 5 -edge-cut $\nabla\left(F_{0}-x\right)$ is not trivial, and $F_{0}-x$ 
is always 2-connected from Theorem 2.5. By Lemma 2.6 we have $p_{5}=k_{3}+1$ for the fullerene patch $F_{0}-x$, which implies that $F_{0}$ has at least one pentagon, contradicting that $F_{0}$ is bipartite.

Corollary 3.2. For each cyclic 6 -edge cut $E_{0}$ of a fullerene graph $F$, both components of $F-E_{0}$ have a perfect matching.

Proof. It follows that $F-E_{0}$ has exactly two components from Lemma 2.2 and 3-edgeconnectedness of $F$. Such two components fulfil the conditions of Theorem 3.1 and thus each has a perfect matching.
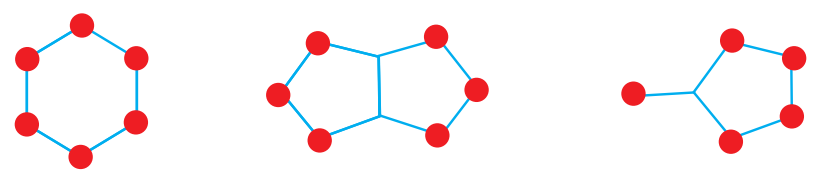

Figure 3: Some nice substructures of fullerene graphs.
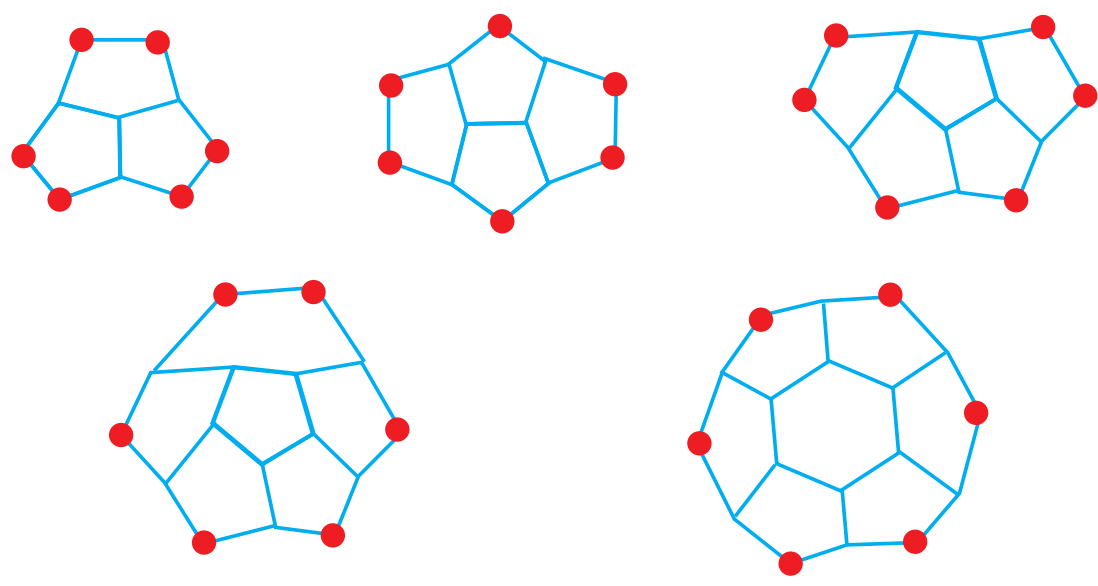

Figure 4: Some nice patches of fullerene graphs with six 2-degree vertices.

From Corollary 3.2 we can find many nice substructures of fullerene graphs, examples of which are shown in Figures 3 and 4. It should be mentioned that the third nice substructure fulvene in Figure 3 has been discovered by Došlić applying 2-extendability of fullerenes [5, 27], and the first one has been proved in investigating $k$-resonance [26, 11]; see the following.

Corollary 3.3 ([26]). Each hexagon of a fullerene graph is resonant.

Corollary 3.4. Each 8-length cycle (if exists) of a fullerene graph bounds a pentalene (a pair of abutting pentagons) and is thus resonant.

Proof. Let $C$ be a 8-length cycle of a fullerene graph $F$. If $F$ has an edge $e$ whose endvertices both lie in $C$ but $e \notin E(C)$, then $e$ is called a chord of $C$. If $C$ has no chords, then the 
eight edges issuing from $C$ can be classified into two edge-cuts of size from 3 to 5 , which lie in the interior and the exterior of $C$ respectively. If one is a 3-edge-cut, then Lemma 2.2 implies that it is trivial, and thus a triangle or quadrilateral appear, a contradiction. If both are 4-edge-cuts, then Lemma 2.3 implies that $F$ has only 12 vertices, also a contradiction. So $C$ must have a chord. Further, this chord and $C$ form a pair of 5 -length cycles sharing this chord, which must bound pentagonal faces of $F$ by Theorem 2.4. That is, $C$ bounds a pentalene and is resonant from Corollary 3.2.

\section{Cyclic 8-edge-cut}

Theorem 4.1. If $E_{0}$ is a cyclic 8-edge-cut of a fullerene graph $F$ and $E_{0}$ is a matching, then $F-E_{0}$ has a perfect matching.

Proof. There exists a nonempty and proper subset $X$ of vertex set $V(F)$ such that $E_{0}=$ $\nabla(X)=[X, \bar{X}]$. Let $F_{0}:=F[X]$ and $\bar{F}_{0}:=F[\bar{X}]$. We claim that both $F_{0}$ and $\bar{F}_{0}$ are connected and $E_{0}$ is a minimal edge-cut. If not, then one of $F_{0}$ and $\bar{F}_{0}$, say $\bar{F}_{0}$, is disconnected. Then $\bar{F}_{0}$ has exactly two components since $F$ is 3 -connected. Since $E_{0}$ is a matching, $F_{0}$ and each component of $\bar{F}_{0}$ have the minimum degree 2 and contain a cycle. So a cyclic edge-cut of at most four edges occurs in $F$, a contradiction. So the claim is verified. Hence each of $F_{0}$ and $\bar{F}_{0}$ has exactly one face of size more than six, which has exactly 8 two-degree vertices on its boundary.

We only show that $F_{0}$ has a perfect matching (the same for $\bar{F}_{0}$ ). If $F_{0}$ has a bridge, then it follows that $F_{0}$ can be obtained from two pentagons by adding one edge between them by Theorems 2.4 and 2.5. In this case $F_{0}$ has a perfect matching. So in the following we always suppose that $F_{0}$ is a patch of $F$. We adopt similar arguments and notations as in the proof of Theorem 3.1 (see Figure 2). It is known that $F_{0}$ has an even number of vertices. Suppose to the contrary that $F_{0}$ has no perfect matchings. By Tutte's theorem we can choose a minimal subset $X_{0} \subset V\left(F_{0}\right)$ satisfying $\alpha:=\operatorname{odd}\left(F_{0}-X_{0}\right) \geq\left|X_{0}\right|+2$.

Let $G_{1}, \ldots, G_{\alpha}$ and $G_{\alpha+1}, \ldots, G_{\alpha+\beta}$ denote respectively the odd components and the even components of $F_{0}-X_{0}$. For $i=1,2, \ldots, \alpha+\beta$, let $m_{i}$ denote the number of edges of $F_{0}$ which are sent to $X_{0}$ from $G_{i}$, and $\gamma_{i}$ (resp. $\gamma_{0}$ ) the number of edges of $F$ from $G_{i}$ (resp. $X_{0}$ ) to the patch $\bar{F}_{0}$. By $\left|\nabla\left(F_{0}\right)\right|=\sum_{i=0}^{\alpha+\beta} \gamma_{i}=8$ and Inequality (3.4), we have

$$
\begin{aligned}
3(\alpha+\beta) & \leq \sum_{i=1}^{\alpha+\beta}\left(m_{i}+\gamma_{i}\right) \\
& \leq 3\left|X_{0}\right|-\gamma_{0}+\sum_{i=1}^{\alpha+\beta} \gamma_{i} \\
& =3\left|X_{0}\right|+8-2 \gamma_{0} \\
& \leq 3 \alpha+2-2 \gamma_{0}
\end{aligned}
$$

which implies that $\beta=0,0 \leq \gamma_{0} \leq 1$, and $\left|X_{0}\right|+2=\alpha$. So the forth equality in Inequality (4.1) holds.

If $\gamma_{0}=1$, then $\left|\left[X-X_{0}, \bar{X}\right]\right|=\sum_{i=1}^{\alpha+\beta} \gamma_{i}=7$ and all equalities in Inequality (4.1) hold. Like the proof of Theorem 3.1 we have that $X_{0}$ is an independent set, $m_{i}+\gamma_{i}=3$ for each $1 \leq i \leq \alpha$ and each $G_{i}$ is a singleton. Hence $F_{0}$ is a bipartite graph. By Lemma 2.6 we have that $F_{0}$ has two three-degree vertices on the boundary of $F_{0}$. That implies that 
$F_{0}$ is just the graph obtained by gluing two hexagons along an edge. So $F_{0}$ has the same cardinalities of two partite sets, which contradicts that $\left|X_{0}\right|+2=\alpha$.

From now on we suppose that $\gamma_{0}=0$. That is, each vertex of $X_{0}$ has degree 3 in $F_{0}$. We claim that second equality in Inequality (4.1) must hold. Otherwise, $F_{0}\left[X_{0}\right]$ has exactly one edge, say $u v$, and the first equality holds, so each $G_{i}$ is a singleton. Without loss of generality, suppose that $y_{1}$ and $y_{2}$ are two neighbors of $u$ other than $v$, and $V\left(G_{1}\right)=\left\{y_{1}\right\}$ and $V\left(G_{2}\right)=\left\{y_{2}\right\}$. Let $X_{0}^{\prime}:=X_{0} \backslash\{u\}$, and $X_{1}:=\left\{u, y_{1}, y_{2}\right\}$. Then $G_{1}^{\prime}:=F_{0}\left[X_{1}\right]$ is a 3 -vertex path obtained by combining $G_{1}$ and $G_{2}$ with vertex $u$. Hence $F_{0}-X_{0}^{\prime}$ has the odd components $G_{1}^{\prime}, G_{3}, \ldots, G_{\alpha}$, and odd $\left(F_{0}-X_{0}^{\prime}\right)=\alpha-1=\left|X_{0}^{\prime}\right|+2$, contradicting the minimality of $X_{0}$.

Hence $X_{0}$ is an independent set of $F_{0}$, and the first inequality is strict. Since for each $1 \leq i \leq \alpha, m_{i}+\gamma_{i}$ is always odd, there exists an $i_{0}$ such that $m_{i_{0}}+\gamma_{i_{0}}=5$ and $m_{i}+\gamma_{i}=3$ for all $i \neq i_{0}$. For convenience, we may suppose that $i_{0}=1$. So $G_{1}$ is an odd component with at least three vertices and $G_{2}, \ldots, G_{\alpha}$ are all singletons. Let $Y_{0}$ denote the set of all singletons $G_{i}(2 \leq i \leq \alpha)$. Then $H:=\left(X_{0}, Y_{0}\right)$ is a bipartite graph as the induced subgraph of fullerene graph $F$.

If $G_{1}$ is a tree, then it is a 2-length path, say $x y z$, since $\nabla\left(G_{1}\right)$ has exactly five edges. For $F_{0}$, by Lemma 2.6 we have $p_{5}=k_{3}-2$. Since $E_{0}$ is a matching, $x$ and $z$ both have neighbors in $X_{0}$, so $\gamma_{1} \leq 3$. The latter implies $\sum_{i=2}^{\alpha} \gamma_{i} \geq 5$. That is, the boundary of $F_{0}$ contains at least 5 two-degree vertices belonging to $Y_{0}$.

We assert that $p_{5} \leq 2$. Since $H$ is bipartite, any pentagon $P$ of $F_{0}$ must intersect $G_{1}$. If $P$ only intersects a vertex of $G_{1}$, say $z$, then $P-z$ is a path of length 3 in $H$ which connects two vertices of $X_{0}$, contradicting that any path between two vertices in the same partite set of a bipartite graph has an even length. Similarly we have that $P$ cannot contain both edges of $G_{1}$. If $F_{0}$ has two distinct pentagons sharing the same edge of $G_{1}$, then one pentagon must have two edges $G_{1}$, a contradiction. So the assertion holds.

By the assertion and $p_{5}=k_{3}-2$ we have $k_{3} \leq 4$. This implies that the boundary of $F_{0}$ has at most 4 vertices in $X_{0}$. Let $C$ be the boundary of $F_{0}$. Then $C-V(C) \cap X_{0}$ has at most $\left|V(C) \cap X_{0}\right|$ components. On the other hand, $C-V(C) \cap X_{0}$ has all singletons in $V(C) \cap Y_{0}$ as components. But $\left|V(C) \cap Y_{0}\right| \geq 5$, contradicting $\left|V(C) \cap X_{0}\right| \leq 4$.

From now on suppose that $G_{1}$ contains a cycle. Then $\nabla\left(G_{1}\right)$ is a cyclic 5-edge-cut of $F$. By Theorem $2.5 \nabla\left(G_{1}\right)$ is a matching and $G_{1}$ is also a patch (precisely, $G_{1}$ is a pentagon or contains a pentacap according as the cyclic 5 -edge-cut $\nabla\left(G_{1}\right)$ is trivial or not), so each vertex of $H$ has degree at least two, and each component of $H$ contains a cycle. If $H$ is disconnected, then $H$ has exactly two components $H_{1}$ and $H_{2}$ since $F_{0}$ is 2-edgeconnected and $\nabla\left(G_{1}\right)$ has exactly five edges. Further, between $G_{1}$ and each $H_{i}$ has at least two edges. So $\nabla\left(G_{1}\right)$ has two consecutive edges along the boundary of $G_{1}$ separately from $G_{1}$ to $H_{1}$ and $H_{2}$. These two edges must be contained in a cycle of length at least 8 bounding a face of $F$, a contradiction. Hence $H$ is connected.

Since $G_{1}$ and $\bar{F}_{0}$ are two connected subgraphs of $F$ with exactly one face of size more than six, there are two possible cases to be considered.

Case 1. $G_{1}$ and $\bar{F}_{0}$ lie in different faces of $H$. Suppose that $G_{1}$ lies in a bounded face $f$ of $H$ and $\bar{F}_{0}$ does in the exterior face of $H$. Then the boundary $\partial f$ of $f$ is a 10-length cycle since 5 neighbors of $G_{1}$ in $H$ belong to $X_{0}$ and are separated by 5 vertices in $Y_{0}$. Hence $F$ is a nanotube with two pentacaps and $F_{0}$ has exactly 6 pentagons. By Lemma 2.6 the boundary of $F_{0}$ has exactly 8 vertices of degree 3 in $F_{0}$. Hence the boundary of $F_{0}$ is an alternating cycle of three-degree and two-degree vertices. But in this nanotube there is only 
10-length cycle as such boundary of a patch, a contradiction.

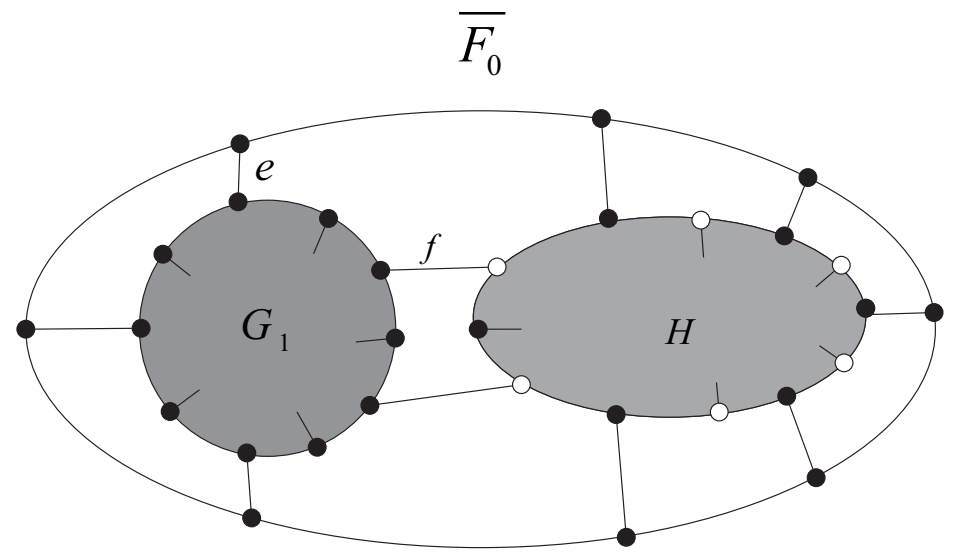

Figure 5: Illustration for Case 2 in the proof of Theorem 4.1 (the vertices in $X_{0}$ are colored white and other vertices black).

Case 2. $G_{1}$ and $\bar{F}_{0}$ lie in the exterior face of $H$. Then the boundary of $F_{0}$ is formed by a path $P$ of $H$ and a path $P_{1}$ of $G_{1}$ and two edges between them. So $0 \leq \gamma_{1} \leq 3$, and there are $8-\gamma_{1}$ two-degree vertices lying on $P$, which belong to $Y_{0}$ and are thus non-adjacent mutually. So there are at least $7-\gamma_{1}$ three-degree vertices in $X_{0}$ on $P$ that can separate them. Since the four end-vertices of $P$ and $P_{1}$ are all of degree three in $F_{0}$, there are at least $11-\gamma_{1}$ vertices of degree three of $F_{0}$ on the boundary. That is, for $F_{0}, k_{3} \geq 11-\gamma_{1}$. On the other hand, if $G_{1}$ is a pentagon, then $F_{0}$ has at most $5-\gamma_{1}$ pentagons, so $k_{3} \leq 7-\gamma_{1}$ by Lemma 2.6, a contradiction. Otherwise, $\nabla\left(G_{1}\right)$ is a non-trivial cyclic 5 -edge-cut and $F_{0}$ has exactly 6 pentagons. Hence, by Lemma 2.6 we have that for $F_{0}, k_{3}=8$. So $\gamma_{1}=3$. Take two consecutive edges $e$ and $f$ of $\nabla\left(G_{1}\right)$ along the boundary of $G_{1}$ separately from $G_{1}$ to $\bar{F}_{0}$ and $H$. Since $\nabla\left(G_{1}\right)$ is a non-trivial cyclic 5 -edge-cut, by Theorem 2.5 we have that $e$ and $f$ have non-adjacent end-vertices in $G_{1}$. So these two edges belong to a cycle of length at least 7 bounding a face of $F$ (see Figure 5). But this is impossible.

From Theorem 4.1 we further find many nice substructures of fullerene graphs, which are listed in Figure 6. In particular, the first one is the naphthalene (a pair of abutting hexagons), whose boundary is a resonant cycle of length 10 .

Corollary 4.2. Any adjacent hexagons of a fullerene graph form a nice substructure, and the boundary (10-length cycle) is thus resonant.

However, not all 10-length cycles of fullerene graphs are resonant. For example, see Figure 1. The following corollary gives a criterion for a 10-length cycle of a fullerene graph to be resonant.

Corollary 4.3. A 10-length cycle $C$ of a fullerene graph $F$ is resonant if and only if it bounds either the naphthalene or the second patch in Figure 4.

Proof. The sufficiency is immediate from Corollaries 3.2 and 4.2. So we only consider the necessity. Suppose that 10-length cycle $C$ of a fullerene graph $F$ is resonant. Let $F_{0}$ be 

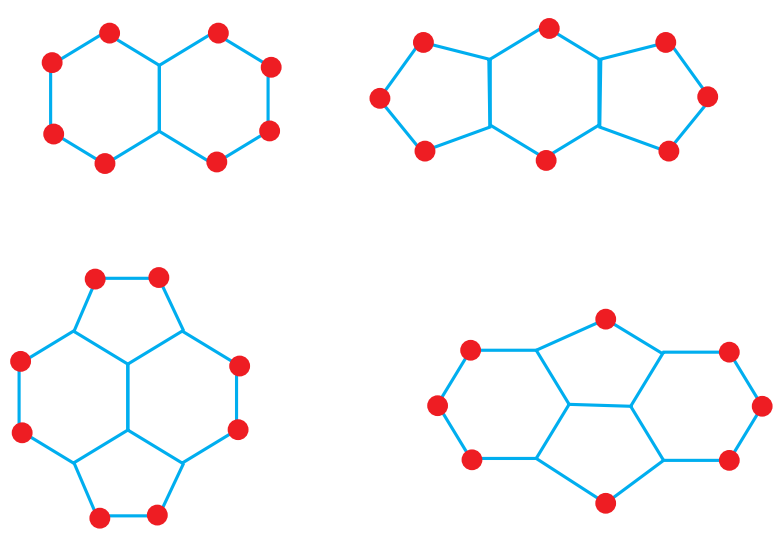

Figure 6: Some nice patches of fullerene graphs with eight 2-degree vertices.

the patch of $F$ bounded by 10-length cycle $C$ with $p_{5} \leq 6$. So $F_{0}$ has an even number of vertices, and we can have that $k_{3}$ and $k_{2}$ both are even. By Lemma 2.6 we have $p_{5}=2 k_{3}-4$ and $2 \leq k_{3} \leq 5$. The possible values of $k_{3}$ are 2 and 4 . If $k_{3}=2$, then $C$ bounds a pair of adjacent hexagons. If $k_{3}=4$, then $F_{0}$ has exactly two vertices in the interior of $C$ which are adjacent by Lemma 2.3. In fact, $F_{0}$ is the second patch in Figure 4.

\section{References}

[1] J. Aihara, Bond resonance energy and verification of the isolated pentagon rule, J. Am. Chem. Soc. 117 (1995), 4130-4136, doi:10.1021/ja00119a029.

[2] J. Aihara, Graph theory of aromatic stabilization, Bull. Chem. Soc. Japan 89 (2016), 14251454, doi:10.1246/bcsj.20160237.

[3] V. Andova, F. Kardoš and R. Škrekovski, Mathematical aspects of fullerenes, Ars Math. Contemp. 11 (2016), 353-379, doi:10.26493/1855-3974.834.b02.

[4] J. Bornhöft, G. Brinkmann and J. Greinus, Pentagon-hexagon-patches with short boundaries, European J. Combin. 24 (2003), 517-529, doi:10.1016/s0195-6698(03)00034-9.

[5] T. Došlić, On some structural properties of fullerene graphs, J. Math. Chem. 31 (2002), 187195, doi:10.1023/a:1016274815398.

[6] T. Došlić, Cyclical edge-connectivity of fullerene graphs and (k,6)-cages, J. Math. Chem. 33 (2003), 103-112, doi:10.1023/a:1023299815308.

[7] P. W. Fowler and D. E. Manolopoulos, An Atlas of Fullerenes, Clarendon Press, Oxford, 1995.

[8] P. W. Fowler, S. Nikolić, R. De Los Reyes and W. Myrvold, Distributed curvature and stability of fullerenes, Phys. Chem. Chem. Phys. 17 (2015), 23257-23264, doi:10.1039/c5cp03643g.

[9] B. Grünbaum and T. S. Motzkin, The number of hexagons and the simplicity of geodesics on certain polyhedra, Canad. J. Math. 15 (1963), 744-751, doi:10.4153/cjm-1963-071-3.

[10] W. C. Herndon, Resonance energies of aromatic hydrocarbons: Quantitative test of resonance theory, J. Am. Chem. Soc. 95 (1973), 2404-2406, doi:10.1021/ja00788a073.

[11] T. Kaiser, M. Stehlík and R. Škrekovski, On the 2-resonance of fullerenes, SIAM J. Discrete Math. 25 (2011), 1737-1745, doi:10.1137/10078699x. 
[12] F. Kardoš and R. Škrekovski, Cyclic edge-cuts in fullerene graphs, J. Math. Chem. 44 (2008), 121-132, doi:10.1007/s10910-007-9296-9.

[13] A. R. Khamatgalimov and V. I. Kovalenko, Molecular structures of unstable isolated-pentagonrule fullerenes $\mathrm{C}_{72}-\mathrm{C}_{86}$, Russ. Chem. Rev. 85 (2016), 836-853, doi:10.1070/rcr4571.

[14] H. W. Kroto, The stability of the fullerenes $\mathrm{C}_{n}$, with $n=24,28,32,36,50,60$ and 70, Nature 329 (1987), 529-531, doi:10.1038/329529a0.

[15] H. W. Kroto, J. R. Heath, S. C. O’Brien, R. F. Curl and R. E. Smalley, C60: Buckminsterfullerene, Nature 318 (1985), 162-163, doi:10.1038/318162a0.

[16] K. Kutnar and D. Marušič, On cyclic edge-connectivity of fullerenes, Discrete Appl. Math. 156 (2008), 1661-1669, doi:10.1016/j.dam.2007.08.046.

[17] L. Lovász and M. D. Plummer, Matching Theory, AMS Chelsea Publishing, Providence, Rhode Island, 2009, doi:10.1090/chel/367, corrected reprint of the 1986 original.

[18] Z. Qi and H. Zhang, A note on the cyclical edge-connectivity of fullerene graphs, J. Math. Chem. 43 (2008), 134-140, doi:10.1007/s10910-006-9185-7.

[19] M. Randić, Conjugated circuits and resonance energies of benzenoid hydrocarbons, Chem. Phys. Lett. 38 (1976), 68-70, doi:10.1016/0009-2614(76)80257-6.

[20] M. Randić, Aromaticity and conjugation, J. Am. Chem. Soc. 99 (1977), 444-450, doi:10.1021/ ja00444a022.

[21] A. Sanz Matías, R. W. A. Havenith, M. Alcamí and A. Ceulemans, Is $\mathrm{C}_{50}$ a superaromat? Evidence from electronic structure and ring current calculations, Phys. Chem. Chem. Phys. 18 (2016), 11653-11660, doi:10.1039/c5cp04970a.

[22] T. G. Schmalz and D. J. Klein, Fullerene structures, in: W. E. Billups and M. A. Ciufolini (eds.), Buckminsterfullerenes, VCH Publishers, New York, chapter 4, pp. 83-101, 1993.

[23] T. G. Schmalz, W. A. Seitz, D. J. Klein and G. E. Hite, Elemental carbon cages, J. Am. Chem. Soc. 110 (1988), 1113-1127, doi:10.1021/ja00212a020.

[24] W. T. Tutte, The factorization of linear graphs, J. London Math. Soc. 22 (1947), 107-111, doi:10.1112/jlms/s1-22.2.107.

[25] Q. Yang, H. Zhang and Y. Lin, On the anti-forcing number of fullerene graphs, MATCH Commun. Math. Comput. Chem. 74 (2015), 673-692, http://match.pmf.kg.ac.rs/ electronic_versions/Match74/n3/match74n3_673-692.pdf.

[26] D. Ye, Z. Qi and H. Zhang, On $k$-resonant fullerene graphs, SIAM J. Discrete Math. 23 (2009), 1023-1044, doi:10.1137/080712763.

[27] H. Zhang and F. Zhang, New lower bound on the number of perfect matchings in fullerene graphs, J. Math. Chem. 30 (2001), 343-347, doi:10.1023/a:1015131912706. 